DOI 10.18699/PlantGen2019-211

\title{
$\beta$-glucan elicitor from Schizophyllum commune induces expression of defense genes and protective effect against Phytopthora blight disease of pepper
}

\author{
Yu Hae-Lin ${ }^{1}$, Kang Kwon Kyoo ${ }^{2,3}$, Kang Hee-Wan ${ }^{1,2,3 *}$ \\ ${ }^{1}$ Graduate School of Future Convergence Technology, Hankyong National University, Ansung, Korea \\ ${ }^{2}$ Department of Horticultural Life Science, Hankyong National University, Ansung, Korea \\ ${ }^{3}$ Institute of Genetic Engineering, Hankyong National University, Ansung, Korea \\ *e-mail:kanghw2@hknu.ac.kr
}

Mushroom, Schizophyllum commune is a white wood-rotting fungi and produce a large of $\beta$-glucan on culturing. The $\beta$-glucan has industrially been used as cosmetics and medicines for enhancing immunity. This study was to investigate the availability of culture filtrate of $S$. commune (Sc-cf) on controlling Phytopthora blight disease of pepper. Sc-cf was precipitated by absolute ethanol and the precipitant and supernatant fractions inhibited mycelial growth of different phytopathogenic fungi including Phytophthora capsici, Rhizoctonia Solani, Pythium ultimum, Botrytis cinerea and Colletotrichum acutatum. The high content $(16.88 \mathrm{~g} / 100 \mathrm{~g})$ of $\beta$-glucan was detected in the precipitant, polysaccharide. The Sc-polysaccharide suppressed Phytophthora blight disease of pepper seedlings more than $60 \%$. In quantitative real-time PCR, the gene expression of CaBPRl (PR protein 1), CaBGLU ( $\beta$-1,3-glucanase), CaPR-4 (PR protein 4), and CaPR-10 (PR protein 10) were significantly induced on the Sc-polysaccharide and DL- $\beta$-aminobutyric acid (BABA) treated pepper leaves. In addition, the salicylic acid (SA) content was also increased in the Sc-polysaccharide treated pepper samples. These results suggest that $\beta$-glucan from $S$. commune can be used as an elicitor for the control of Phytopthora blight disease of pepper. 